\title{
Mapping Medical Disasters: Ebola Makes Old Lessons, New
}

\author{
Tom Koch, PhD
}

\section{ABSTRACT}

Disaster medicine is characterized by shortages of everything but patients. There are never enough beds, equipment, personnel, or supplies. In the 2014 Ebola epidemic, another scarcity was maps. The need for maps of the affected areas, and the ways the maps were used, serve to emphasize the way maps have always served in both disaster medicine and public health preparedness. Those lessons are reviewed here in the context of the Ebola epidemic. (Disaster Med Public Health Preparedness. 2015;9:66-73)

Key Words: disaster medicine, disaster planning, disease outbreaks, medical cartography, spatial epidemiology

F or researchers, what is now officially known as the West African Ebola virus disease epidemic has been something of a boon. "It's a very serious epidemic, but everything has a silver lining," the Director of Development Research for the African Development Bank told reporters. ${ }^{1}$ In response to the epidemic, millions of dollars of research-related dollars have flowed into the region. And, too, popular and professional attention was focused on countries that are too often ignored. For medical and public health professionals, however, it is difficult to see the epidemic as anything but a human disaster. For personnel in the field and for those who support them, the real issue is what can be learned to prevent future disasters and to ensure that future infectious disease outbreaks can be better contained and, where containment is impossible, more effectively managed.

There are lessons to be learned. For example, in the best of conditions and with fulsome international effort, it will take at least 12 to 18 months before a vaccine can be designed, tested, and approved for general use. Even if a vaccine or prophylactic treatment is available, it will require months before contracts are signed for ramped-up production of medicines that must then be distributed to regional centers for eventual delivery to patients living in far-flung, isolated regions.

In the interim, older disaster medicine and public health protocols must be enacted. This article reviews the utility of medical mapping as one of those areas of traditional response. The argument will be that field mapping as a surveillance system, and more generally as a tool of field medicine and public health, is a critical instrument in the identification, investigation, and treatment of isolated outbreaks threatening epidemic expansion.

\section{MEDICAL MAPPING}

Since the late 17th century, mapping has been a critical tool used by physicians and public health officials confronting local outbreaks, regional epidemics, and broad pandemic events. ${ }^{2}$ Since the late 18th century, mapping has also served as a fundamental medium for the presentation and testing of theories of disease incidence and transmission. ${ }^{3}$ In the early 20th century, disease mapping was widely taught as a principal technique in "sanitary science," the forerunner of modern epidemiology and public health. ${ }^{4}$ Its prominence continued through most of the century.

In recent years, however, medical mapping has been assumed by some to serve illustratively but not substantially in disease management and study. It has been used primarily as at best a pictorial summation of the analytic calculations underlying a primarily statistical "spatial epidemiology." ${ }^{5}$ Much of that work has focused on a general consideration of disease incidence on global and regional scales. ${ }^{6,7}$ Other studies have used mapping as an investigatory medium for the analysis of specific disease ecologies. ${ }^{8}$

Whatever their scale or specific subject, all maps perform two critical functions, one existential and the other geographic. ${ }^{9}$ All maps argue the existence of something in a place. "This (cholera, Ebola, tuberculosis) is here (this street, town, county, country)." Second, individual cases (the rows in a dataset) are 
presented as similarly symbolized members of a single event class. This transforms a set of distinct occurrences into parts of a single thing (The Epidemic).

The central thesis of medical mapping is that disease events have a spatial structure based upon the locational incidence of mortality and morbidity in an environment whose individual constituents promote or retard the progress of a specific disease. ${ }^{10}$ By defining different events as members of a single class, and positioning them in relation to other event classes, a set of dynamic relational structures with explanatory and prescriptive potential is enacted.

It is not simply the commonality of events of a similar nature but the relations between them (usually based upon density and distance) that is important. For example, maps of cholera incidence typically will include a second event class of local water sources, one or more of which is the suspected source of an outbreak. In public health mapping, classes of disease incidence are mapped with classes of available clinic and hospital resources if it is a question of resource availability and allocation.

\section{Ebola Mapping}

Perhaps the earliest map of Ebola was included in a March 24 report issued by the Republic of Guinea's Health Ministry. ${ }^{11}$ The map identified 4 adjacent prefectures, distinguished by red hatching, in which Ebola had been confirmed: Guéckédou, where the majority of deaths had occurred; Macenta; Kissidougou; and N'Zérékoré. In the map the names of neighboring countries (Liberia, Mali, Senegal, and Sierra Leone) were lettered but otherwise not distinguished. The map thus argued a limited outbreak in 4 provinces of 1 country. The potential for disease diffusion to neighboring countries was implicitly denied in this manner. The map conformed to the assumption that, like previous outbreaks beginning in the 1970s, this one would be spatially contained in nonmetropolitan, interior areas.

In its March 27, 2014, report, the World Health Organization (WHO) similarly mapped all national prefectural boundaries, coloring red those reporting active Ebola cases (Figure 1). ${ }^{12}$ That map included the coastal prefecture of Conakry and its port city of the same name where 4 cases were confirmed and a fifth was suspected. That brought the total number of confirmed Ebola cases to 103. The possibility of other new infections was being investigated, at that time, in only 2 adjacent provinces, which were colored pink.

For a medical geographer, the WHO map, but not the Ministry of Health's map, would have raised a warning flag. A slow diffusion from Guéckédou to neighboring districts would be expected and could potentially be managed with local containment and treatment programs. But a hotspot on the nation's capital, Conakry, promised the likelihood of an expansive epidemic. Localized in central provinces with sparse populations, the Ebola outbreak would, presumably, be containable and short lived. But an outbreak in Conakry, the nation's capital with an estimated population of nearly 2 million persons, ${ }^{13}$ was something else. Here was the possibility of a large outbreak in an urban population in a city with maritime and other connections to cities in the region and the world.

The questions the first Conakry cases raised were thus exigent. Were they an independent, unrelated outbreak or was the source the Guéckédou outbreak? If the latter, as seemed likely, were the Conakry cases the result of personto-person transmission or something else (perhaps a food source)? If the outbreak in Conakry was expansive rather than independent, if Ebola was traveling, where else might it soon appear?

If, in retrospect, the epidemic that resulted was an "avoidable crisis," as some have argued, ${ }^{14}$ here was where it might have been stopped. Early symptoms of epidemic expansion posted in national prefectural maps were ignored, or at the least, not sufficiently credited. Experts assumed reasonably, but in the end inaccurately, what the March WHO map denied: Ebola was always a highly localized, short-term, typically rural event. A complex of factors ranging from regional poverty, a minimal health infrastructure, poor communication between attending agencies, and an environment conducive to viral expansion all contributed to the outbreak. But the failure to see in this and subsequent early disease maps the epidemiological signs of expansion delayed a series of potentially effective interventions.

\section{Epidemic Expansion}

As the epidemic matured, the scale of the maps expanded beyond the Republic of Guinea to permit inclusion of cases confirmed in adjacent countries. By the summer of 2014, the resolution of these maps increased to permit the mapping of relative incidence in various cities in the region (Figure 2). In these "dot maps" the size of the circle was based on the number of reported cases. In some maps, pie charts were used to distinguish between "confirmed," "probable," and "suspected" cases in a city or town. In official reports by the WHO and others, graphs of the increasing epidemic curvein cities and across the region-were included based on the same data as the maps. Eventually, maps of the regional epidemic included a third event class in which hospitals and medical facilities were included. These did not, however, include institutional capacity (number of beds, doctors, and nurses, etc) and patient loads. Had those data been included, the inadequacy of existing medical resources-local and international—would have been immediately evident.

These dot maps, which were widely reproduced in international newspapers, served as evidence of a serious, expansive epidemic. 


\section{FIGURE 1}

March 27, 2014, World Health Organization Map of Ebola in Guinea.

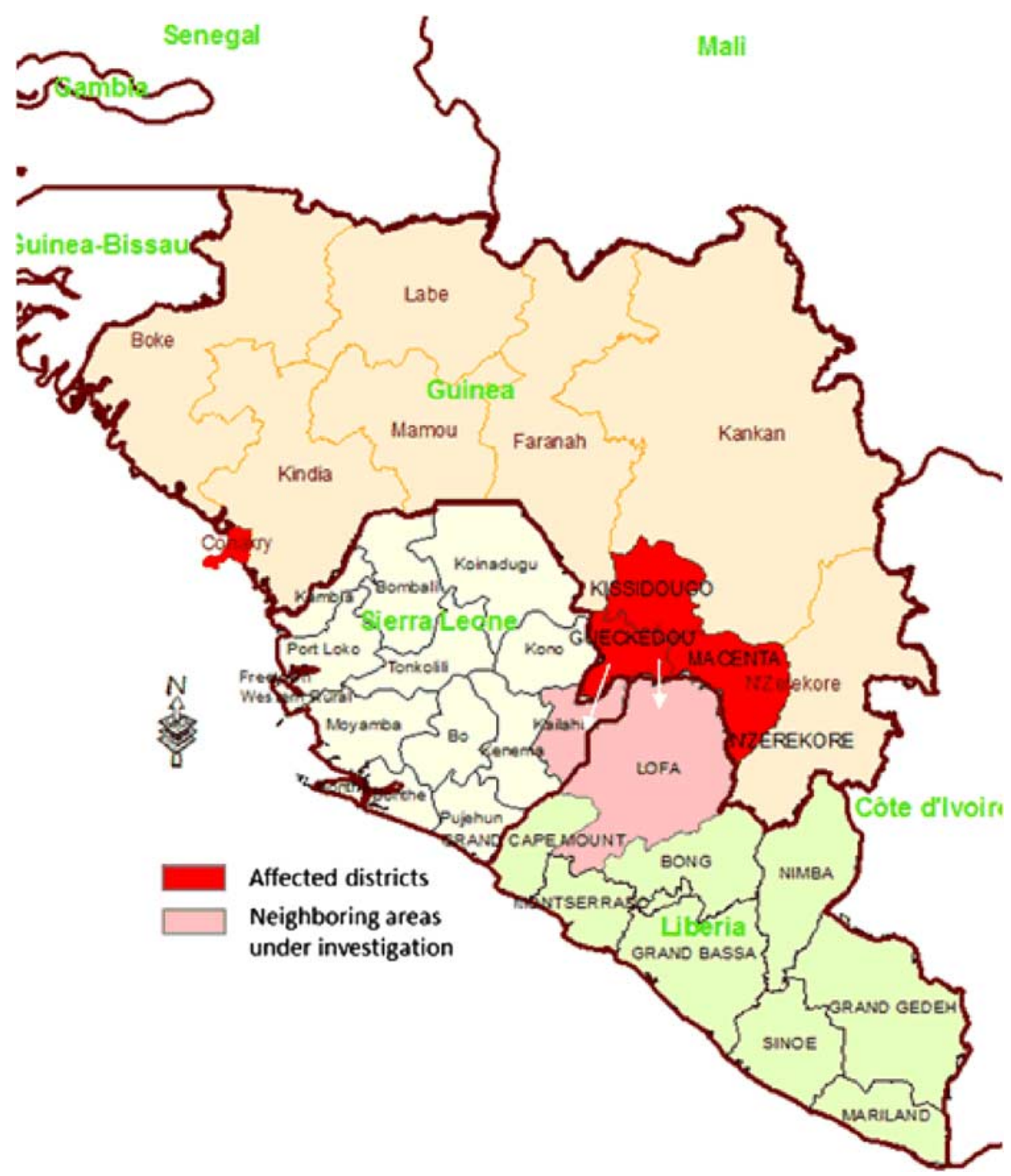

The map presented a localized outbreak in 4 provinces in the Republic of Guinea and, separately, the port area of Conakry. Source: World Health Organization Regional Office for Africa website.

http://www.afro.who.int/en/clusters-a-programmes/dpc/epidemic-a-pandemic-alert-and-response/outbreak-news/4069-ebola-virus-diseaseguinea-27-march-2014.html. ${ }^{12}$

Expansion into Conakry and Freetown (Sierra Leone) and Monrovia (Liberia)-larger port cities-signaled potential pandemic expansion. The maps argued, simply, "this (epidemic) is here and it might soon be everywhere." Increasingly, maps of potential pandemic occurrence, and then international cases involving former health workers, were published.

\section{Local Mapping}

When the first cases of Ebola hemorrhagic fever were reported in March 2014, Swiss-based Médicine sans Frontièrs (MSF) quickly dispatched an epidemiological team to Guéckédou, Guinea, the epicenter of what was to become a regional epidemic. The MSF team included a geographic information systems (GIS) specialist who, over an 8-week period, created 109 maps of local roads, landmarks, and villages, in Guéckédou prefecture. ${ }^{16}$ In a region without existing maps of affected towns and villages, this was critical. Without these maps, tracking the incidence of Ebola across the villages and towns of a region was difficult. Approximately $60 \%$ of the GIS specialist's time therefore was spent on producing way-finding maps of the roads and villages in the region; the remainder 
FIGURE 2

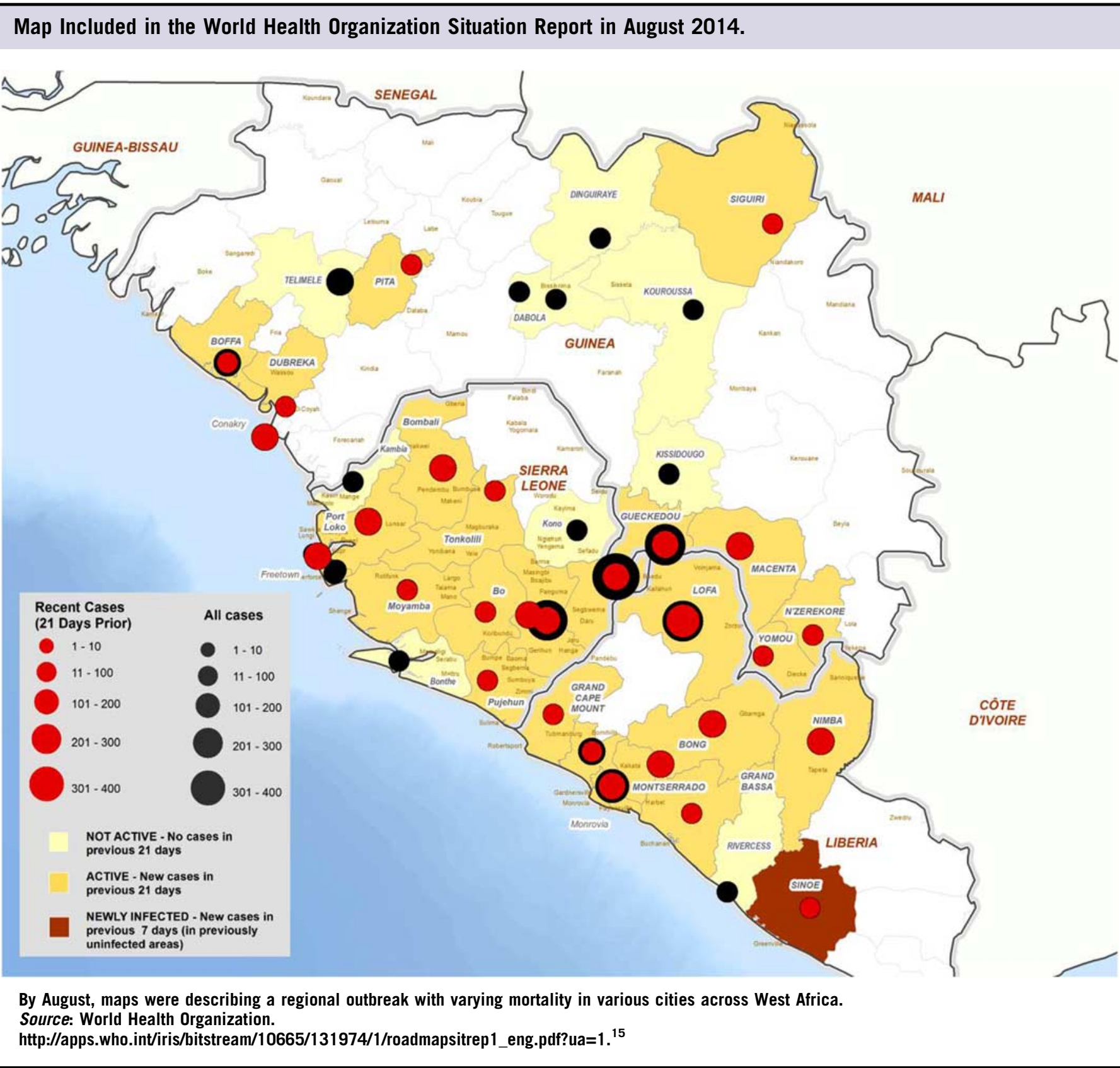

was used to create maps that addressed public health concerns over the location of water, hygiene, and sanitation teams. Only a few maps were made to serve epidemiological investigations.

In recent decades, mapping has been transformed from a manual to a digital skill with the use of computer-based GIS programs. Before the MSF team's departure, a general map of the Guéckédou region was traced based on satellite images of the region. In this process, a satellite image of the target area is displayed in a program permitting streets, buildings (homes, hospitals, schools, etc), and biogeographic elements (lakes, streams, etc) to be constructed as separate digital event classes. The result is at once spatially precise and easily modified when new data become available (for example, patient location). These satellite-based computer maps are typically composed of lines (for streets), points (precise locations), and polygons (biogeographic and political boundaries) and can be easily printed or digitally distributed.

The resulting way-finding maps were critical for foreign field workers unfamiliar with the region. Without them, getting from medical sites to local villages would have been impossible. Also, the maps permitted a picture of the spatial 


\section{FIGURE 3}

\section{A 1912 Map Attempting to Identify the Chain of Diffusion in a Polio Outbreak in Mason City, lowa.}

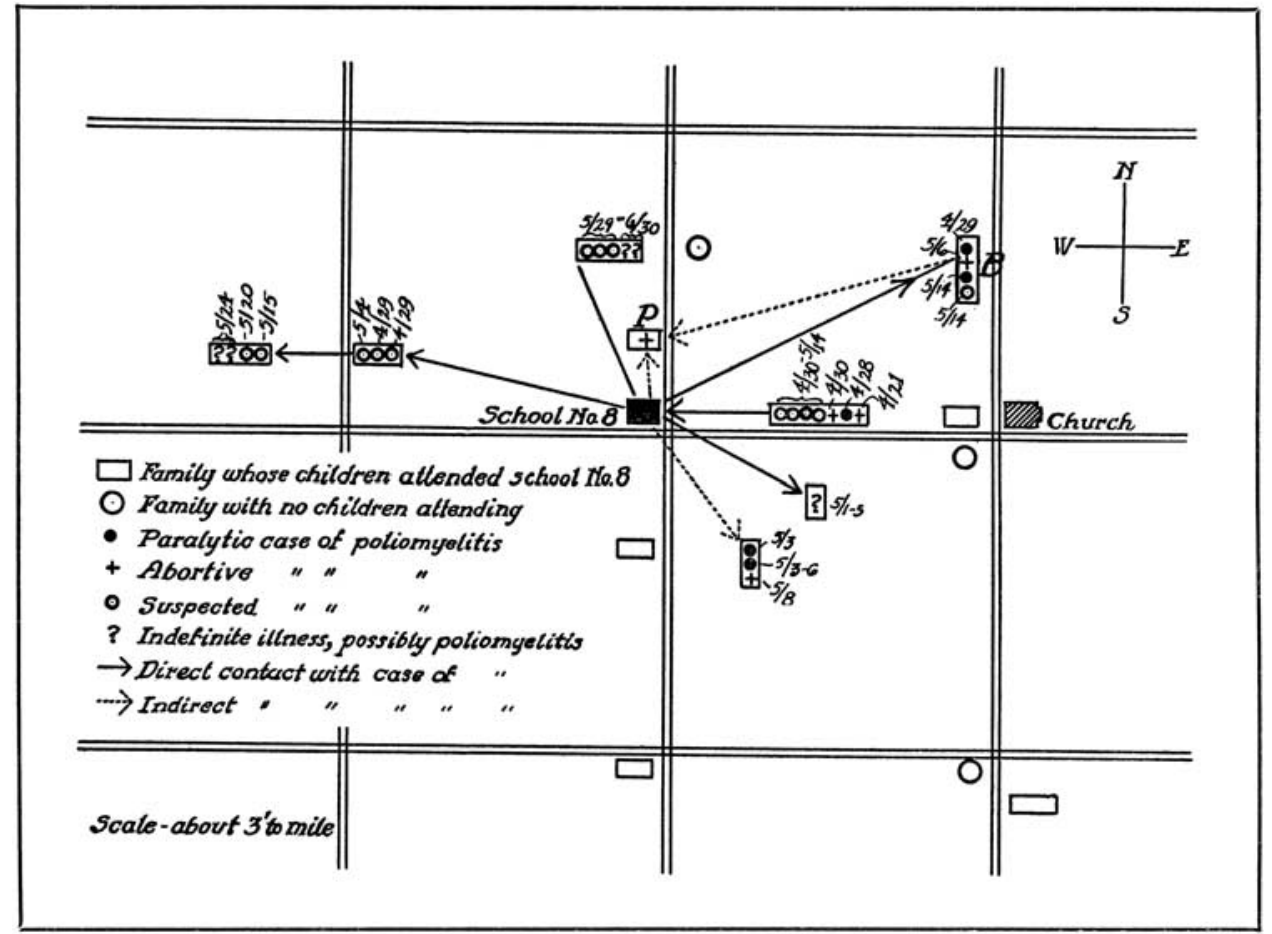

Source: Frost WH. Hygienic Laboratory Bulletin. 2012;90:9-105; 234-52. ${ }^{20}$

spread of the epidemic to be ascertained. Within the target prefecture, for example, 14 separate villages were named "Bendou." Identifying which Bendou was the home of a specific patient was potentially critical to those tracing the chain of disease transmission and to others attempting to understand the general structure of the district epidemic. ${ }^{16}$ "The challenge is good information, because information helps tell us where the disease is, how it's spreading and where we need to target our resources," a United Nations emergency response official told reporters in October 2014. ${ }^{17}$ "Unfortunately, we don't have good data from a lot of areas. We don't know exactly what is happening."17 Mapping was a critical medium in which data on "what is happening" could be collected, organized, analyzed, and presented. Because local and foreign workers often spoke different languages, maps served as a lingua franca promoting shared knowledge among those who otherwise had difficulty communicating.

\section{HOT Mapping}

As the epidemic expanded, detailed and accurate maps of newly infected towns and villages across the infected territory were similarly unavailable. Innovatively, MSF and Red Cross International decided to crowdsource the task of mapping these newly infected, previously unmapped towns and villages. ${ }^{18}$ The idea had been tried before but never at this scale. Hundreds of international volunteers were invited to join the "missing maps" project organized by Humanitarian OpenStreetmap (HOT). Participants registered, logged in online, and were directed to satellite maps of towns or villages where maps were urgently required by ground personnel. In some countries, "HOT parties" were organized for volunteers. ${ }^{19}$

"Firstly," the website of the program instructed, "we need to trace the features of the target location. Using aerial photographs as a backdrop, we can literally trace road networks, buildings and landmarks to build a line drawing of the area." Once a contributor's work was saved to the website, it was integrated automatically into the cooperatively constructed evolving map. Local personnel then added the names of buildings, neighborhoods, lakes, roads, etc, to the digital construct. Those were then uploaded to volunteers who entered them into the map's final version. As a result, what otherwise would have taken weeks of individual labor required only a few days per map.

\section{Epidemic Mapping}

Inclusion of a GIS expert resulted from an earlier MSF study describing epidemiology as "the domain where GIS can bring the most positive evolution." " Yet, the potential contribution of mapping to the medical effort was hampered by ignorance 


\section{Territorial Range of Fruit Bats Implicated in the Ebola Epidemic of 2014.}

The extent of the map is roughly congruent with the area most affected by the virus Source: Pigot DM, Golding N, Mylne A, et al. ELife. 2014;10.7554/eLife.04395. http://elifesciences.org/content/early/2014/09/05/eLife.04395. ${ }^{23}$

about the uses of maps in an epidemic environment. "Most MSF staff know very little about GIS in general, let alone how a GIS officer could support them."16 As a result, the GIS team member spent relatively little time contributing to epidemiological studies.

That MSF team members knew little about the use of maps in epidemic studies and public health initiatives was unfortunate. Beginning with the mapping of yellow fever in late 18th century cities, maps have served as a vehicle through which an infectious chain of transmission can be traced in an effort to uncover the source of an outbreak. ${ }^{3}$ Figure 3 is a typical illustration of this kind of mapping by W. H. Frost, a US epidemiologist investigating a poliomyelitis outbreak in Mason City, Iowa, in 1912. ${ }^{20}$ The mapped approach was based on techniques developed for single-source, bacterial disease outbreaks such as cholera and typhoid fever in the 19th century.
In the current Ebola outbreak, transmission mapping at the beginning of the outbreak might have alerted field medical personnel that the outbreak that they thought was contained in May was in fact expanding. Mapping of affected persons and their travels during the period of disease incubation almost certainly would have described what was only later recognized. Citizens of Meliandou, where the epidemic's 2013 index case was eventually located, and Guéckédou in Guinea lived in a porous border region across which Kissi-speaking persons regularly traveled into Liberia and Sierra Leone. The effort to contain the disease in Guinea was therefore doomed without a cooperative effort by all 3 countries to contain an outbreak in a shared region. "The most tragically missed opportunities stemmed from the poor flow of information about who was infected and whom they might have exposed."21 That failure of information was more geographic than medical, and thus potentially reportable via mapping of local travel patterns. 


\section{Investigatory Mapping}

Medical mapping has served as a means to both develop and test theories about the environmental origins of infectious disease outbreaks. In this case, increasing attention was paid to the possibility that the outbreak was promoted not only by person-to-person transmission but also through the food chain in which wild animals ("bush meat") served as a local protein source. ${ }^{22}$ Of special interest was the prevalence of fruit bats, a primary protein source in local diets. ${ }^{23}$ Bats have been previously implicated in the transmission of several diseases, including Ebola, MERS, SARS, and Marburg hemorrhagic fever. ${ }^{24}$ Thus, by the autumn of 2014 some disease ecologists were mapping the natural territory of bats both as a way of considering their engagement in this epidemic and in a first assessment of the potential of the Ebola virus to spread to other areas in Africa with robust, shared bat populations.

Figure 4 by Pigot et $\mathrm{a}^{23}$ is one of a series of maps of bat species suspected as potential West African Ebola reservoirs. The dots on the map locate observation points used to identify the species' spatial range. Coloration, yellow to green, describes the relative probability of the presence of the target species, in this case the little colored fruit bat (Myonycteris torquata).

\section{Public Health Preparedness}

Epidemics and pandemics are composed of a set of localized outbreaks in which scarcity typically reigns. There is never enough equipment: beds, medicines, or ventilators are in short supply; doctors and nurses are overwhelmed by patient volumes. In localized events, those shortages typically are quickly relieved through the support of neighboring health districts or federal assistance. This was the case in the United States, for example, following Hurricane Katrina. ${ }^{25}$ That becomes less possible, however, in a disease event affecting a broad geography when shortages of equipment and personnel become regional rather than local. This was especially true during the West African Ebola epidemic where existing health infrastructures were, at the start, at best minimal. ${ }^{26}$

Mapping provides an essential medium in which at-risk populations can be first estimated and then matched with probable care needs early in an outbreak. Algorithms exist for the estimation of disease transmission rates, incubation periods, and the reproduction number of specific viruses, including Ebola. ${ }^{27}$ Other algorithms permit rapid population estimates based on the density of buildings and, if available, housing density in specific communities. Knowing where existing cases are, and the extent of public health resources available for their treatment, permits public health responders to not only provide more efficiently what is needed but estimate what likely will be needed in different disease scenarios.

\section{DISCUSSION}

It is easy to look back and see what, during a crisis, was unclear. None of this is to be taken as criticism of the extraordinary efforts of MSF, Red Cross, or WHO personnel. Nor is it meant as criticism of the efforts of medical or public health officials in the affected countries. Rather it is to suggest that lessons may be learned to limit potential epidemics in the future and to better care for both those affected and the personnel who care for them.

A signal lesson of local, national, and international responses to the 2014 Ebola epidemic was the failure to immediately recognize the dynamic and expansive nature of the event. Some blamed the WHO and the budget cuts that had decimated its roster of experienced personnel. ${ }^{28}$ Others blamed systemic regional poverty, the effects of deforestation, and a history of conflicts. ${ }^{29}$ These were critical and their importance is not to be underestimated.

Instead, I argue here that the failure to contain the outbreak at its onset resulted in part from its expansion being unnoticed, unseen. Workers on the ground warned of an emerging epidemic, but without detailed and shared maps, those warnings apparently were discounted. The paucity of maps appears to have contributed to a failure by both local authorities and the international community to "connect the dots" and then react aggressively and proactively to dynamic outbreaks in various locales with, in retrospect, obvious epidemic potential.

Among the lessons of the Ebola outbreak, therefore, is the need for rigorous medical mapping as soon as the presence of potentially contagious outbreaks is noted. Accompanying these incidence maps can and should be others describing medical resources, populations, and, at another scale, proximity to neighboring, potentially at-risk populations. Those data would serve to create a better early warning system of outbreaks threatening epidemic status. With that early warning, localized interventions can be planned.

This will require the engagement of persons trained in mapping the epidemiology of disease events and the public health issues surrounding them. It will require those persons' knowledgeable participation in systems of international, regional, and local disease planning and surveillance. Doing this work in map-poor environments with few clinical resources and a range of cultures and languages presents specific challenges. A future article will detail the nature and potential of field mapping in such environments as a practical adjunct to medical care and public health initiatives.

If Ebola taught us nothing else, it served as a reminder-if one is truly needed-that local and regional health crises are always potentially international in nature. Yesterday's outbreak "there" may become ours tomorrow "here." Communicable and infectious diseases spread across the globe in predicable ways and, in the map, the necessity for cooperative responses to disease events can be seen, assessed, and collectively confronted. 


\section{About the Author}

Department of Geography (Medical), University of British Columbia, 1984 West Mall, Vancouver, British Columbia, Canada.

Correspondence and reprint requests to Tom Koch, clo 136 Hammersmith Ave, Toronto, Ontario, Canada M4E 2W6 (e-mail: tomkoch@kochworks.com).

\section{Acknowledgment}

The author wishes to express his gratitude to the journal editors and the anonymous peer reviewer whose comments were critical to the development of this article.

Published online: February 9, 2015.

\section{REFERENCES}

1. Clark P. Funding Research in Africa. The Scientist. 2015;29(1):28-29. http://www.the-scientist.com/?articles.view/articleNo/41683/title/FundingResearch-in-Africa/. Accessed January 20, 2014.

2. Koch T. Disease Maps: Epidemics on the Ground. Chicago, IL: University of Chicago Press; 2012:51-58.

3. Koch T. Cartographies of Disease: Maps, Mapping, and Medicine. Redlands, CA: ESRI Press; 2005:26-33.

4. Sedgwick WT. Principles of Sanitary Science and the Public Health, with Special Reference to the Causation and Prevention of Infectious Diseases, 4th Ed. New York, NY: MacMillan; 1911.

5. Elliott P, Wartenberg D. Spatial epidemiology: current approaches and future challenges. Environ Health Perspect. 2004;112(9):998-1006.

6. Hay SI, George DB, Moyes CL, Brownstein JS. Big data opportunities for global infectious disease surveillance. PLoS Med. 2013;10(4). doi: 10.1371/journal.pmed.1001413.

7. Hay SI, Battle KB, Pigott DM, et al. Global mapping of infectious disease. Philos Trans R Soc London B Biol Sci. 2013:368. http://royalsocietypublishing. org/content/royptb/368/1614/20120250.full.pdf. Accessed January 23, 2015.

8. Liu Y, Jiang S, Liu Y, et al. Spatial epidemiology and spatial ecology study of worldwide drug-resistant tuberculosis. Int J Health Geogr. 2011;10:50. http://www.biomedcentral.com/content/pdf/1476-072X-1050.pdf. Accessed November 20, 2014.

9. Wood D, Fels J. The Natures of Maps: Cartographic Constructions of the Natural World. Chicago, IL: University of Chicago Press; 2008:xvi-xvii.

10. Haggett P. The Geographical Structure of Epidemics. New York, NY: Oxford University Press; 2000.

11. Republic of Guinea, Ministry of Health. Info Sur la Fievre Hemorragique a Virus Ebola en Guinee-Conakry. http://photos.state.gov/libraries/guinea/ 231771/PDFs/infofieverebolafrench03052014.pdf. Published April 5, 2014. Accessed January 23, 2015.

12. Ebola virus disease, Guinea (Situation as of 27 March 2014). World Health Organization Regional Office for Africa website. http://www. afro.who.int/en/clusters-a-programmes/dpc/epidemic-a-pandemic-alert-andresponse/outbreak-news/4069-ebola-virus-disease-guinea-27-march-2014.html. Published March 27, 2014. Accessed January 24, 2015.
13. U.S. Relations with Guinea. Fact Sheet. Bureau of African Affairs, U.S. Department of State. http://www.state.gov/r/pa/ei/bgn/2824.htm. Published January 23, 2014. Accessed February 4, 2014.

14. Jeremy J, Farrar J, Piot P. The Ebola emergency-immediate action, ongoing strategy. N Engl J Med. 2014;371(16):1545-1546.

15. WHO: Ebola Response Roadmap Situation Report 1. World Health Organization. http://apps.who.int/iris/bitstream/10665/131974/1/ roadmapsitrep1_eng.pdf?ua $=1$. Published August 29, 2014. Accessed December 19, 2014.

16. Lüge T. GIS Support for the MSF Ebola response in Guinea in 2014, Case Study. Geneva, Switzerland: Médicins sans Frontières, Logistics Department; 2014. http://reliefweb.int/report/world/gis-support-msf-ebolaresponse-guinea-2014-case-study. Published July 15, 2014. Accessed November 13, 2014.

17. Muhumuza R. Poorly tracked Ebola cases hampering efforts, says UN. AP/Toronto (Canada) Star. http://www.thestar.com/news/world/2014/10/ 28/poorly_tracked_ebola_cases_hampering_efforts_says_un.html. Published October 28, 2014. Accessed December 10, 2014.

18. Missing Maps Project. Medecins Sans Frontieres website. http://www.msf. org.uk/missing-maps-project. Accessed January 23, 2015.

19. Mapping Parties. Openstreetmap.org. http://wiki.openstreetmap.org/wiki/ Mapping_parties. Accessed December 13, 2014.

20. Frost WH. Epidemiologic studies of acute anterior poliomyelitis. Hygienic Laboratory Bulletin. 2012;90(9-105):234-252.

21. Sacks K, Fink S, Belluck P, et al. Ebola's deadly escape. The New York Times. December 30, 2014:D2.

22. Funk S, Piot P. Mapping Ebola in wild animals for better disease control. ELife. 2014;3:e04565. http://elifesciences.org/content/3/e04565. Accessed September 21, 2014.

23. Pigot DM, Golding N, Mylne A, et al. Mapping the zoonotic niche of Ebola virus disease in Africa. ELife. 2014; 10.7554/eLife.04395. http:// elifesciences.org/content/early/2014/09/05/eLife.04395. Accessed November 24, 2014.

24. Aberlin MB. Bad raps: understanding animal diseases-for their sake and ours. The Scientist. 2014;28(12):13. http://www.the-scientist.com/? articles.view/articleNo/41484/title/Bad-Raps/. Accessed December 15, 2014.

25. Fink S. Five Days at Memorial: Life and Death in a Storm-Ravaged Hospital. New York, NY: Crown; 2013.

26. Young J. 'Grim scene' from Ebola outbreak show need to rebuild West African health care." Toronto (Canada) Star. December 12, 2014. http:// www.thestar.com/news/world/2014/12/11/grim_scenes_from_ebola_outbreak_ show_need_to_rebuild_west_african_health_care.html. Accessed December 14, 2014.

27. Dietz K. The estimation of the basic reproduction number for infectious diseases. Stat Methods Med Res. 1993;2(1):23-41.

28. Fink S. Cuts at W.H.O. hurt response to Ebola Crisis. New York Times. September 3, 2014. http://www.nytimes.com/2014/09/04/world/africa/ cuts-at-who-hurt-response-to-ebola-crisis.html?_r=1. Accessed December 12, 2014.

29. Bausch DG, Schwarz L. Outbreak of Ebola virus disease in Guinea: where ecology meets economy. PLOS Negl Trop Dis. 2014;9(7). http://www. plosntds.org/article/info\%3Adoi\%2F10.1371\%2Fjournal.pntd.0003056. Accessed December 15, 2014. 